\title{
Stochastic Hybrid Dynamic Multicultural Social Networks
}

\author{
Kristina B. Hilton, G.S. Ladde \\ Department of Mathematics and Statistics, University of South Florida, Tampa, FL, United States; \\ khilton@mail.usf.edu; gladde@usf.edu
}

\begin{abstract}
In this work, we investigate the cohesive properties of a stochastic hybrid dynamic multi-cultural network under random environmental perturbations. By considering a multi-agent dynamic network, we model a social structure and find conditions under which cohesion and coexistence is maintained using Lyapunov's Second Method and the comparison method. In this paper, we present a prototype illustration that exhibits the significance of the framework and approach. Moreover, the explicit sufficient conditions in terms of system parameters are given to exhibit when the network is cohesive both locally and globally. The sufficient conditions are algebraically simple, easy to verify, and robust. Further, we decompose the cultural state domain into invariant sets and consider the behavior of members within each set. We also analyze the degree of conservativeness of the estimates using Euler-Maruyama type numerical approximation schemes based on the given illustration.
\end{abstract}

Keywords: Multi-agent Network; Cohesiveness; Lyapunov Second Method; Invariant Sets.

\section{Introduction}

The aim of this work is to explore and extend the cohesive properties of a dynamic network of multiagents/members with a desired minimum safe distance between the members of the network [1-3] under the influence of both continuous and discrete-time stochastic perturbations. Dynamic network models play an important role in a variety of modeling applications. For example, economics, finance, engineering, management sciences, and biological networks have considered such large-scale dynamic models to investigate connectivity, stability, dynamic reliability, and convergence [4-7].

One of the concepts studied using a dynamic social network is that of consensus [8-11]. In such models, the conditions under which a group collectively comes to an agreement on an issue under consideration are studied. Another question of interest for such a network is when the group may divide into subgroups with an agreement reached within the subgroup but never reaching a consensus at an overall group level. Most of the work done in these areas look to develop consensus seeking algorithms and consider long term stability of the network in consideration [12-15].

The concepts of cohesion, coordination, and cooperation within a group are often multi-faceted, dynamic and complex, but are important concepts when trying to better understand how nations or communities function [16]. We seek to better understand the group dynamics of such a society in order to create policies and practices that encourage a sense of community among individuals from a variety of cultural backgrounds. 

and Communications, Volume 5 No. 5, October (2017); pp: 1-23

In fact, we systematically initiated the study of this issue $[2,3]$ to better understand the social dynamics of a group seeking to find such a balance under the influence of both continuous and discrete-time deterministic and stochastic perturbations. In doing so, we are interested in better understanding the cohesive properties of a multi-cultural social network. In this work, we further extend the developed results in the framework of hybrid stochastic dynamic model for which we explore the features of the network. By considering a hybrid dynamic [17], we are able to consider the impact that events both from external and internal stochastic fluctuations coupled with an intervention process on the network have on the cultural dynamics. The presented work is used to exhibit the quantitative and qualitative properties of the network. Further, the techniques used are computationally attractive and algebraically simple relating with the underlying network parameters. This feature plays an important role for planning and decision processes.

In Section 2, we present a general problem under consideration and the underlining assumptions. We then present an illustration of such a network in Section 3 to exhibit the role and scope of the underlying complexity with the simplicity without loss of generality. Using an appropriate energy function and the comparison method, upper and lower estimates on cultural states are established in Section 4. In Section 5 , the long-term behavior of the solutions to the comparison equations are examined and we explore the study of the cultural state invariant sets in the context of the illustration presented in Section 3. In Section 6 , we use numerical simulations to model the network and to better understand to what extent the analytically developed estimates in Section 5 are feasible. Overall, the presented results are conservative but are reliable and robust.

\section{Problem Formulation}

The network consists of $m$ agents whose position at time $t$ is represented by $x_{i}(t), i \in I(1, m)=\{1,2, \ldots, m\}$, with $x_{i}(t) \in \square^{n}$. In our model, this vector does not represent a geographical location but rather a cultural position of the ith member. That is to say, the vector $x_{i}$ is a numerical representation of the ith member's beliefs or background on certain cultural or ethnic practices relevant to the network under study. Further, we assume that $\xi_{i j}, i, j \in I(1, m)$ is a normalized Wiener process such that $\xi_{i j}=\xi_{j i}$ and for $j \neq k, \xi_{i j}$ and $\xi_{i k}$ are independent. We then consider a system of ItôDoob type stochastic system of differential equations that describes the cultural state dynamic process:

$$
\left\{\begin{aligned}
& d x_{i}=\sum_{j=1}^{m} f\left(t, x_{i}, x_{i}-x_{j}, k-1\right) d t+\sum_{j \neq i}^{m} \sigma\left(t, x_{i}-x_{j}, k-1\right) d \xi_{i j}(t), \\
& \Delta x_{i}^{k}=I\left(x_{i}^{k-1}\left(t_{k}^{-}, t_{k-1}, x_{i}^{k-1}\right), k\right), \quad x_{i}^{0}\left(t_{0}\right)=x_{i}^{0},
\end{aligned}\right.
$$

for $\left(t, x_{i}\right) \in\left[t_{k-1}, t_{k}\right) \times \square^{n}$ and $k \in I(1, \infty)$, where $x_{i}, x_{j} \in \square^{n}$ are continuous time dynamic states; $i, j \in I(1, m) ; f$ and $\sigma$ are drift and diffusion rate coefficient functions, respectively; and $\Delta x_{i}^{k}=x_{i}^{k}-x_{i}^{k-1}$, where $I$ in (1) stands for a discrete time intervention dynamic process. We will also make the following assumptions:

Assumption $\mathrm{H}_{1}$ : For 
○ $x_{i}^{k-1}\left(t_{k-1}\right)=x_{i}^{k-1}$ is an $n$-dimensional initial cultural state random vector defined on the complete probability space $(\Omega, F, P)$ and $k \in I(0, \infty)$ at the $k$ th intervention time;

$\circ \quad x_{i}^{k-1}$ and $\xi_{i j}(t)$ are mutually independent for each $t_{k-1} \leq t<t_{k}$ for $i \neq j, i, j \in I(1, m)$ and $k \in I(0, \infty)$;

○ For $i, j \in I(1, m), \xi_{i}(t)=\left(\xi_{i 1}, \xi_{i 2}, \ldots, \xi_{i j} \ldots, \xi_{i m}\right)^{T}$ is a $m$-dimensional normalized Wiener process of independent increments for $i \in I(1, m)$;

$\circ \xi_{i j}$ are $F_{t}$-measurable for all $t \geq t_{0}$ and $\xi_{i j}(t+h)-\xi_{i j}(t)$ is independent of $F_{t}$, where $F_{t}$ represents an increasing family of the smallest sub- $\sigma$ algebra of $F$, i.e. $F_{s} \subset F_{t}$ if $t_{0}<s<t$;

○ $x_{i}\left(t_{0}\right)$ is $F_{t_{0}}$ measurable;

○ $\left\{t_{k}\right\}_{k=1}^{\infty}$ is a sequence of intervention time, and $t_{k} \rightarrow \infty$ as $k \rightarrow \infty$;

$\circ f$ and $\sigma$ are defined on: $\square_{+} \times \square^{n} \times \square^{n} \times I(1, \infty)$ into $\square^{n}$ and continuous on $\left[t_{k-1}, t_{k}\right) \times \square^{n} \times \square^{n}$ for each $(t, x, y) \in\left[t_{k-1}, t_{k}\right) \times \square^{n} \times \square^{n}$;

$\circ \quad f$ and $\sigma$ satisfy for each $k \in I(1, \infty)$ and for each $(t, x, y, k) \in\left[t_{k-1}, t_{k}\right) \times \square^{n} \times \square^{n} \times I(1, \infty)$

$$
\begin{aligned}
& f(t, x, y, k-1) \rightarrow f\left(t_{k}^{-}, x, y, k-1\right) \\
& \sigma(t, x, y, k-1) \rightarrow \sigma\left(t_{k}^{-}, x, y, k-1\right)
\end{aligned}
$$

as $t \rightarrow t_{k}^{-}$;

○ $\quad I: \square^{n} \times I(1, \infty) \rightarrow \square^{n}$ is a Borel measurable discrete time intervention function.

It is assumed that the initial value problem (1) for the system of stochastic differential equations has a solution process.

We wish to investigate the stochastic cohesive property of such a network. Further, we will explore the behavior of a member of the network based on the cultural state distance between a network member cultural state and the cultural state center of the network.

Below, we state a few definitions with regard the quantitative and qualitative behavior of the cultural network.

Definition 1: Let $r_{1}$ and $r_{2}$ be non-negative random functions for $t \in\left[t_{k-1}, t_{k}\right), k \in I(1, \infty)$ such that $r_{1} \leq r_{2}$. We say that a stochastic multicultural dynamic network is:

i. locally cohesive with probability 1 , if for any $N \in F_{t}$ such that $P(N)=0, N \subset \Omega$ and for all $t \in\left[t_{k-1}, t_{k}\right]$,

$$
r_{1}(t) \leq\left\|x_{i}^{k-1}(t)-x_{j}^{k-1}(t)\right\| \leq r_{2}(t),
$$


for all $i, j \in I(1, m)$;

ii. locally cohesive in probability, if for all $i, j \in I(1, m), t \in\left[t_{k-1}, t_{k}\right)$, and any $0<\epsilon<1$

$$
P\left(\left\{\Omega:\left\|x_{i}^{k-1}(t)-x_{j}^{k-1}(t)\right\|<r_{1}(t) \text { or }\left\|x_{i}^{k-1}(t)-x_{j}^{k-1}(t)\right\|>r_{2}(t)\right\}\right)<\epsilon,
$$

for all $i \in I(1, m)$;

iii. locally cohesive in pth mean, if for all $i, j \in I(1, m)$ and $t \in\left[t_{k-1}, t_{k}\right)$

$$
E\left[r_{1}(t)\right] \leq E\left[\left\|x_{i}^{k-1}(t)-x_{j}^{k-1}(t)\right\|^{p}\right] \leq E\left[r_{2}(t)\right],
$$

for all $i \in I(1, m)$.

If (i.), (ii.), or (iii.) exist for all $t \in\left[t_{0}, \infty\right)$, we say the network is globally cohesive with probability 1 , in probability or in $p$ th mean respectively.

Definition 2: We say that a stochastic multicultural dynamic network

i. locally reaches a consensus with probability 1 , if there exists $N \subset F$ such that $P(N)=0$ for all $\omega \in \Omega \backslash N$,

$$
\lim _{t \rightarrow \infty}\left\|x_{i}^{k-1}(t)-\bar{x}^{k-1}\right\|=0,
$$

for $k \in I(1, \infty)$ and all $i, j \in I(1, m)$;

ii. locally reaches a consensus in probability, if for $\epsilon>0$ and $t \in\left[t_{k-1}, t_{k}\right), k \in I(1, \infty)$

$$
\lim _{t \rightarrow \infty} P\left(\left\{\left\|x_{i}^{k-1}(t)-\bar{x}^{k-1}\right\|>\epsilon\right\}\right)=0,
$$

for all $i \in I(1, m)$;

iii. locally reaches a consensus in the pth mean, if for $t \in\left[t_{k-1}, t_{k}\right), k \in I(1, \infty)$

$$
\lim _{t \rightarrow \infty} E\left[\left\|x_{i}(t)-\bar{x}\right\|^{p}\right]=0 \text {, }
$$

for all $i \in I(1, m)$.

If (i.), (ii.), or (iii.) exist for all $t \in\left[t_{0}, \infty\right)$, we say the network is reaches a global consensus with probability 1 , in probability or in $p$ th mean respectively.

Let $x_{i}^{k-1}$ and $x_{j}^{k-1}$ be cultural state random vectors for $i, j \in I(1, m)$ and $k \in I(1, \infty)$. For $t \in\left[t_{k-1}, t_{k}\right)$, we define the relative cultural state affinity with probability 1 sense by

$$
\left\|x_{i}^{k-1}(t)-x_{j}^{k-1}(t)\right\| .
$$


We note that the relative cultural state affinity in the a.s. sense exists as $\|\bullet\|$ is Borel measurable.

\section{Prototype Dynamic Model}

Let us define a prototype multicultural network dynamic model under the stochastic environmental perturbations described by the Itô-Doob type stochastic system of differential equations

$$
\left\{\begin{aligned}
d x_{i}^{k-1} & =\left[a_{k-1} \sum_{j=1}^{m} x_{i j}^{k-1}-q_{k-1}\left\|x_{i}^{k-1}-\bar{x}^{k-1}\right\|^{2} \sum_{j=1}^{m} x_{i j}^{k-1}\right. \\
& \left.+b_{k-1} \sin \left\|x_{i}^{k-1}-\bar{x}^{k-1}\right\| \sum_{j=1}^{m} x_{i j}^{k-1} \exp \left[-\frac{\left\|x_{i j}^{k-1}\right\|^{2}}{c_{k-1}}\right]\right] d t \\
& +\beta_{k-1} \sin \left\|x_{i}^{k-1}-\bar{x}^{k-1}\right\| \sum_{j=1}^{m} x_{i j}^{k-1} \exp \left[-\frac{\left\|x_{i j}^{k-1}\right\|^{2}}{c_{k-1}}\right] d \xi_{i j}, \\
x_{i}^{k} & =\left(1+\delta_{i}^{k-1}\right) x_{i}^{k-1}\left(t_{k}^{-}, t_{k-1}, x_{i}^{k-1}\right), \quad x_{i}^{0}\left(t_{0}\right)=x_{i}^{0},
\end{aligned}\right.
$$

for $t \in\left[t_{k-1}, t_{k}\right), k \in I(0, \infty)$ and where $a_{k-1}, q_{k-1}, b_{k-1}, c_{k-1}$ and $\beta_{k-1}$ are positive real numbers, and

$$
x_{i j}^{k-1}=x_{i}^{k-1}-x_{j}^{k-1},
$$

We note that the solution process $x_{i}$ of (10) is defined by

$$
x_{i}\left(t, t_{0}, x_{i}^{0}\right)= \begin{cases}x_{i}^{0}\left(t, t_{0}, x_{i}^{0}\right) & t_{0} \leq t<t_{1}, \\ x_{i}^{1}\left(t, t_{1}, x_{i}^{1}\right) & t_{1} \leq t<t_{2}, \\ \vdots & \vdots \\ x_{i}^{k-1}\left(t, t_{k-1}, x_{i}^{k-1}\right) & t_{k-1} \leq t<t_{k}, \\ \vdots & \vdots\end{cases}
$$

Here, $\bar{x}^{k-1}$ is the center of the multicultural dynamic system (1) defined by:

$$
\bar{x}^{k-1}=\frac{1}{m} \sum_{j=1}^{m} x_{j}^{k-1}(t), \quad t \in\left[t_{k-1}, t_{k}\right),
$$

and note that by substituting for $x_{i}^{k-1}$ by $\bar{X}^{k-1}$ into (10), we have 


$$
\begin{aligned}
d \bar{x}^{k-1} & =\left[a_{k-1} \sum_{j=1}^{m}\left(\bar{x}^{k-1}-x_{j}^{k-1}\right)-q_{k-1}\left\|\bar{x}^{k-1}-\bar{x}^{k-1}\right\|^{2} \sum_{j=1}^{m}\left(\bar{x}^{k-1}-x_{j}^{k-1}\right)\right. \\
& \left.+b_{k-1} \sin \left\|\bar{x}^{k-1}-\bar{x}^{k-1}\right\| \sum_{j=1}^{m}\left(\bar{x}^{k-1}-x_{j}^{k-1}\right) \exp \left[-\frac{\left\|\bar{x}^{k-1}-x_{j}^{k-1}\right\|}{c_{k-1}}\right]\right] d t \\
& +\beta_{k-1} \sin \left\|\bar{x}^{k-1}-\bar{x}^{k-1}\right\| \sum_{j=1}^{m}\left(\bar{x}^{k-1}-x_{j}^{k-1}\right) \exp \left[-\frac{\left\|\bar{x}^{k-1}-x_{j}^{k-1}\right\|^{2}}{c_{k-1}}\right] d \xi_{\bar{x}^{k-1} j}, \\
& =a_{k-1} m \bar{x}^{k-1}-a_{k-1} \sum_{j=1}^{m} x_{j}^{k-1} \\
& =a_{k-1} m \bar{x}^{k-1}-a_{k-1} m \bar{x}^{k-1} \\
& =0
\end{aligned}
$$

for $t \in\left[t_{k-1}, t_{k}\right), k \in I(1, \infty)$. Thus $\bar{x}^{k-1}$ defined in (13) is a stationary center of the multicultural dynamic network on each interval $\left[t_{k-1}, t_{k}\right)$. We define the transformation $z_{i}^{k-1}=x_{i}^{k-1}-\bar{x}^{k-1}$ and observe that $x_{i j}^{k-1}=Z_{i}^{k-1}-Z_{j}^{k-1}=Z_{i j}^{k-1}$. Then the transformed network dynamic model corresponding to (10) is reduced to:

$$
\left\{\begin{aligned}
d z_{i}^{k-1} & =\left[a_{k-1} m z_{i}^{k-1}-q_{k-1}\left\|z_{i}^{k-1}\right\|^{2} m z_{i}^{k-1}+b_{k-1} \sin \left\|z_{i}^{k-1}\right\| \sum_{j=1}^{m} z_{i j}^{k-1} \exp \left[-\frac{\left\|z_{i j}^{k-1}\right\|^{2}}{c_{k-1}}\right] d t\right. \\
& +\beta_{k-1} \sin \left\|z_{i}^{k-1}\right\| \sum_{j=1}^{m} z_{i j}^{k-1} \exp \left[-\frac{\left\|z_{i j}^{k-1}\right\|^{2}}{c_{k-1}}\right] d \xi_{i j}, \quad t \in\left[t_{k-1}, t_{k}\right) \\
z_{i}^{k} & =\left(1+\delta_{i}^{k-1}\right) z_{i}^{k-1}\left(t_{k}^{-}, t_{k-1}, z_{i}^{k-1}\right), \quad z_{i}^{0}\left(t_{0}\right)=z_{i}^{0} .
\end{aligned}\right.
$$

The center $\bar{X}^{k-1}$ of the multicultural dynamic model (10) is reduced to the center zero in (15) over each interval $\left[t_{k-1}, t_{k}\right)$ and $k \in I(1, m)$. For each $k \in I(1, \infty), a_{k}, b_{k}, c_{k}, q_{k}$ represent the weight of the social moderation attractiveness $\left(q_{k}\right)$, the repulsive forces $\left(a_{k}\right)$, the rate of decay of the long-range attractiveness $\left(c_{k}\right)$, and the long-range attractiveness $\left(b_{k}\right)$ between individual members and social groups. Further, the parameter $\beta_{k}$ characterizes the random environmental perturbations. It exhibits both attractive and repulsive forces that are centered at the center of the network. The magnitude of the 
repulsive forces over $\left[t_{k-1}, t_{k}\right]$ are described by $a_{k-1} m\left\|z_{i}^{k-1}\right\|$ and the magnitude of the long range deterministic attractive forces are characterized by

$$
b_{k-1}\left\|\sum z_{i j}^{k-1} \exp \left[-\frac{\left\|z_{i j}^{k-1}\right\|^{2}}{c}\right]\right\| \text {. }
$$

Further, $\sin \left\|z_{i}^{k-1}\right\|$ is the sine-cyclical influence of the ith member's relative distance to the center of the network. The stochastic term represents the environmental influence due to long-range attractive forces. In particular, in the case of a multi-cultural network, the noise captures the uncertainty generated due to the membership interactions and deliberations under the influence of the long-range cultural forces.

We remark that the solution process of (15) can be re-casted as (12). In order to study the multicultural dynamics (15), we use Lyapunov's Second Method in conjunction with the comparison method [18]. These methods are computationally attractive and provide a means of better understanding the movement and behavior of the state memberships of the network. By utilizing these methods, we are able to establish conditions for which we have both upper and lower estimates on the members' cultural state positions on the interval $\left[t_{k-1}, t_{k}\right)$ for $k \in I(1, m)$. In this work, we assume that all inequalities are with probability 1.

\section{Upper and Lower Comparison Equations}

Using Lyapunov's Second Method and differential inequalities, we first seek a function $r_{k-1}\left(t, t_{k-1}, u_{k-1}\right)$ such that

$$
\left\|z_{i}^{k-1}(t)\right\| \leq r_{k-1}\left(t, t_{k-1}, r_{k-1}\right), \quad t \in\left[t_{k-1}, t_{k}\right) .
$$

From Definition 1, relation (17) generates a concept of a locally upper-cohesive cultural network in the almost sure sense on the $k-1$ th interval for $k \in I(1, \infty)$.

To this end, for $t \in\left[t_{k-1}, t_{k}\right)$ let us choose an energy function $V_{k-1}$ as:

$$
V_{k-1}\left(z_{i}^{k-1}\right)=\left\|z_{i}^{k-1}\right\|=\left(\left(z_{i}^{k-1}\right)^{T} z_{i}^{k-1}\right)^{\frac{1}{2}} .
$$

We have previously shown $[2,3]$ that the differential of $V_{k-1}$ in the direction of the vector field represented by (15) is 


$$
\begin{aligned}
d V_{k-1} & =\frac{\left(z_{i}^{k-1}\right)^{T} d z_{i}^{k-1}}{\left\|z_{i}^{k-1}\right\|}+\frac{1}{2}\left[\frac{\left(d z_{i}^{k-1}\right)^{T} d z_{i}^{k-1}}{\left\|z_{i}^{k-1}\right\|}-\frac{\left(\left(z_{i}^{k-1}\right)^{T} d z_{i}^{k-1}\right)^{2}}{\left\|z_{i}^{k-1}\right\|^{3}}\right] \\
& =\frac{\left(z_{i}^{k-1}\right)^{T} \sum_{j=1}^{m} \phi_{2}\left(z_{i j}^{k-1}\right) d \xi_{i j}}{\left\|z_{i}^{k-1}\right\|}+L V\left(z_{i}^{k-1}\right) d t
\end{aligned}
$$

where

$$
\begin{aligned}
& \phi_{1}\left(z_{i}^{k-1}\right)=a_{k-1} m z_{i}^{k-1}-q_{k-1}\left\|z_{i}^{k-1}\right\|^{2} m z_{i}+b_{k-1} \sin \left\|z_{i}^{k-1}\right\| \sum_{j=1}^{m} z_{i j}^{k-1} \exp \left[-\frac{\left\|z_{i j}^{k-1}\right\|^{2}}{c_{k-1}}\right] \\
& \phi_{2}\left(z_{i j}^{k-1}\right)=\beta_{k-1} \sin \left\|z_{i}^{k-1}\right\| z_{i j}^{k-1} \exp \left[-\frac{\left\|z_{i j}^{k-1}\right\|^{2}}{c_{k-1}}\right]
\end{aligned}
$$

and

$$
\begin{aligned}
& L V_{k-1}\left(z_{i}^{k-1}\right)=\frac{\left(z_{i}^{k-1}\right)^{T} \phi_{1}\left(z_{i}^{k-1}\right) d t}{\left\|z_{i}^{k-1}\right\|}+\frac{\sum_{j=1}^{m} \phi_{2}^{T}\left(z_{i j}^{k-1}\right) \phi_{2}\left(z_{i j}^{k-1}\right)}{2\left\|z_{i}^{k-1}\right\|}-\frac{\left(\left(z_{i}^{k-1}\right)^{T} \sum_{j=1}^{m} \phi_{2}\left(z_{i j}^{k-1}\right)\right)^{2}}{2\left\|z_{i}^{k-1}\right\|^{3}} \\
& =\left[a_{k-1} m\left\|z_{i}^{k-1}\right\|-q_{k-1} m\left\|z_{i}^{k-1}\right\|^{3}+\frac{b_{k-1} \sin \left\|z_{i}^{k-1}\right\|}{\left\|z_{i}^{k-1}\right\|} \sum_{j=1}^{m}\left(z_{i}^{k-1}\right)^{T} z_{i j}^{k-1} \exp \left[-\frac{\left\|z_{i j}\right\|^{2}}{c_{k-1}}\right]\right. \\
& +\frac{\beta_{k-1}^{2} \sin ^{2}\left\|z_{i}^{k-1}\right\| \sum_{j=1}^{m}\left(z_{i j}^{k-1}\right)^{T} z_{i j}^{k-1} \exp \left[-\frac{2\left\|z_{i j}^{k-1}\right\|^{2}}{c_{k-1}}\right]}{2\left\|z_{i}^{k-1}\right\|} \\
& \left.-\frac{\beta_{k-1}^{2} \sin ^{2}\left\|z_{i}^{k-1}\right\| \sum_{j=1^{m}}\left(\left(z_{i}^{k-1}\right)^{T} z_{i j}^{k-1}\right)^{2} \exp \left[-\frac{2\left\|z_{i}^{k-1}\right\|^{2}}{c_{k-1}}\right]}{2\left\|z_{i}^{k-1}\right\|^{3}}\right] d t .
\end{aligned}
$$


In the following, we present a result that will be used subsequently.

Lemma 1: Let $V_{k-1}$ be the energy function defined in (18) and $z_{i}^{k-1}$ be a solution of the initial value problem defined in (15). Then, for each $i \in I(1, m), k \in I(1, \infty)$, and $t \in\left[t_{k-1}, t_{k}\right)$,

$$
E\left[V_{k-1}\left(z_{i}^{k-1}(t+\Delta t)\right)-V_{k-1}\left(z_{i}^{k-1}(t)\right) \mid F_{t}\right]=L V_{k-1}\left(z_{i}^{k-1}(t)\right) \Delta t
$$

where $E$ stands for the expected value.

Proof: For each $k \in I(1, \infty)$, let $z_{i}^{k-1}\left(t, t_{k-1}, z_{i}\left(t_{k-1}\right)\right)$ be the solution process of (15). Let $F_{t}$ be an increasing family of sub $-\sigma$ algebras as previously defined and set

$$
m(t)=E\left[V_{k-1}\left(z_{i}^{k-1}(t)\right) \mid F_{t}\right]=V\left(z_{i}^{k-1}(t)\right),
$$

where the last equality holds as $z_{i}^{k-1}(t)$ is $F_{t}$ measurable. Similarly, we have set

$$
m(t+\Delta t)=E\left[V_{k-1}\left(z_{i}^{k-1}(t+\Delta t)\right) \mid F_{t}\right]
$$

for all $\Delta t>0$ sufficiently small such that $(t+\Delta t) \in\left[t_{k-1}, t_{k}\right)$. We consider

$$
\begin{aligned}
m(t+\Delta t)-m(t) & =E\left[V_{k-1}\left(z_{i}^{k-1}(t+\Delta t)-V_{k-1}\left(z_{i}^{k-1}(t)\right)\right) \mid F_{t}\right] \\
& =E\left[\frac{\partial V_{k-1}}{\partial z}\left(z_{i}^{k-1}(t)\right) \Delta z_{i}^{k-1}(t)+\frac{1}{2} \operatorname{tr}\left(\frac{\partial^{2} V_{k-1}}{\partial z^{2}}\left(\Delta z_{i}^{k-1}(t)\right)\left(\Delta z_{i}^{k-1}(t)\right)^{T}\right) \mid F_{t}\right] \\
& =E\left[d V_{k-1}\left(z_{i}^{k-1}(t)\right) \mid F_{t}\right]
\end{aligned}
$$

This together with (19), yields

$$
\begin{aligned}
m(t+\Delta t)-m(t) & =E\left[L V_{k-1}\left(z_{i}^{k-1}(t)\right) \Delta t \mid F_{t}\right] \\
& =L V_{k-1}\left(z_{i}^{k-1}(t)\right) \Delta t
\end{aligned}
$$

as $Z_{i}^{k-1}(t)$ is $F_{t}$ measurable. We note that for small $\Delta t$, we have

$$
d m(t)=L V_{k-1}\left(z_{i}^{k-1}(t)\right) d t
$$

\subsection{Upper Estimate of $L V_{k-1}\left(z_{i}^{k-1}\right)$}

We seek constraints on the parameters $a_{k-1}, b_{k-1}, c_{k-1}, q_{k-1}$ and $\beta_{k-1}, k \in I(1, \infty)$ for which we have an upper estimate on $V_{k-1}\left(z_{i}^{k-1}\right)$. To this end, imitating the argument made in [3], an upper estimate of $L V_{k-1}$ in $(21)$ is 


$$
\begin{aligned}
L V_{k-1} & \leq q_{k-1} m\left\|z_{i}\right\|\left(\frac{a_{k-1}}{q_{k-1}}\right. \\
& +\frac{\left.4 b_{k-1}(m-1) \sqrt{\frac{c_{k-1}}{2} \exp \left[-\frac{1}{2}\right]+\beta_{k-1}^{2}(m-1) c_{k-1} \exp [-1]}-\left\|z_{i}\right\|^{2}\right)}{4 q_{k-1} m} \\
& \leq q_{k-1} m V_{k-1}\left(\eta_{k-1}^{2}-V_{k-1}^{2}\right) \\
& \leq q_{k-1} m V_{k-1}\left(\eta_{k-1}-V_{k-1}\right)\left(\eta_{k-1}+V_{k-1}\right),
\end{aligned}
$$

where $\eta_{k-1}$ is defined by

$$
\eta_{k-1}=\left(\frac{a_{k-1}}{q_{k-1}}+\frac{4 b_{k-1}(m-1) \sqrt{\frac{c_{k-1}}{2}} \exp \left[-\frac{1}{2}\right]+\beta_{k-1}^{2}(m-1) c_{k-1} \exp [-1]}{4 q_{k-1} m}\right)^{\frac{1}{2}} .
$$

From the inequality (28) utilizing the comparison method [18] and Lemma 1, we establish the following lemma. For each interval $\left[t_{k-1}, t_{k}\right)$ and $k \in I(1, \infty)$, the presented result establishes not only an upper bound but also the locally upper cohesive property almost surely. Hereafter, all inequalities and equalities are assumed to be valid with probability one.

\section{Lemma 2:}

Let $V_{k-1}$ be the energy function defined in (18), $k \in I(1, \infty), t \in\left[t_{k-1}, t_{k}\right)$, and $z_{i}^{k-1}$ be a solution of the initial value problem defined in (15). Let $r_{k-1}(t)$ be the maximal solution [18] of a random initial value problem

$$
d u_{k-1}=\left[q_{k-1} m u_{k-1}\left(\eta_{k-1}-u_{k-1}\right)\left(\eta_{k-1}+u_{k-1}\right)\right] d t, \quad u_{k-1}\left(t_{k-1}\right)=u_{k-1}
$$

where $\eta_{k-1}$ is defined as in (29). For each $V_{k-1}\left(z_{i}^{k-1}\right), i \in I(1, m)$, and $k \in I(1, \infty)$ satisfying the differential inequality (28) and $V_{k-1}\left(z_{i}^{k-1}\left(t_{k-1}\right)\right) \leq u_{k-1}$, it follows that the multicultural dynamic network (10) is upper cohesive on $\left[t_{k-1}, t_{k}\right]$ with probability 1 and

$$
V_{k-1}\left(z_{i}^{k-1}(t)\right) \leq r_{k-1}\left(t, t_{k-1}, u_{k-1}\right)
$$

Proof:

From Lemma 1, (28), and the application of stochastic comparison theorem [18], with probability 1, it follows that 


$$
V_{k-1}\left(z_{i}^{k-1}(t)\right) \leq r\left(t, t_{k-1}, u_{k-1}\right)
$$

when $V_{k-1}\left(z_{i}\left(t_{k-1}\right)\right) \leq u_{k-1}$. As the solution to (30) has an upper bound, the network is upper cohesive almost surely.

Remark 1: For each $k \in I(1, \infty)$, if the solution processes of (15) and (30) have a first moment, then the solution process of (15) is locally upper 1st moment cohesive. Furthermore, under the current inequality, it is indeed locally upper cohesive in the sense of probability.

\subsection{Lower Estimate of $L V_{k-1}\left(z_{i}^{k-1}\right)$}

Next we consider the lower comparison equation. Using Lyapunov's Second Method and differential inequalities, we next seek a function $\rho_{k-1}\left(t, t_{k-1}, u_{k-1}\right)$ such that

$$
\left\|z_{i}(t)\right\| \geq \rho\left(t, t_{k-1}, \rho_{k-1}\right), \quad t \in\left[t_{k-1}, t_{k}\right) .
$$

Again, from Definition 1, relation (33) initiates a notion of a locally lower cohesive cultural dynamic network in the almost sure sense.

Using the energy function defined in (18) and relation (21), for $t \in\left[t_{k-1}, t_{k}\right)$ it follows that

$$
\begin{aligned}
L v_{k-1} \quad & a_{k-1} m V_{k-1}-q_{k-1} m V_{k-1}^{3}-V_{k-1}(m-1) b_{k-1} \sqrt{\frac{c_{k-1}}{2}} \exp \left[-\frac{1}{2}\right] \\
& -\frac{\beta_{k-1}^{2}(m-1) c_{k-1} \exp [-1]}{4} V_{k-1} \\
= & q_{k-1} m V_{k-1}\left(\frac{a_{k-1}}{q_{k-1}} \sqrt{\frac{c_{k-1}}{2}} \exp \left[-\frac{1}{2}\right]+\beta_{k-1}^{2} c_{k-1}(m-1) \exp [-1]\right. \\
4 q_{k-1} m &
\end{aligned}
$$

Assumption $\mathrm{H}_{2}$ : Assume there exists a positive number $\alpha_{k-1}$ such that

$$
\alpha_{k-1} \leq\left(\frac{a_{k-1}}{q_{k-1}}-\frac{4(m-1) b_{k-1} \sqrt{\frac{c_{k-1}}{2}} \exp \left[-\frac{1}{2}\right]+\beta_{k-1}^{2}(m-1) c_{k-1} \exp [-1]}{4 q_{k-1} m}\right)^{\frac{1}{2}} .
$$

From (34), and noticing the fact that assumption $\mathrm{H}_{2}$ implies 


$$
\frac{a_{k-1}}{q_{k-1}}>\frac{4(m-1) b_{k-1} \sqrt{\frac{c_{k-1}}{2}} \exp \left[-\frac{1}{2}\right]+\beta_{k-1}^{2}(m-1) \exp [-1]}{4 q_{k-1} m}
$$

it follows that

$$
L V_{k-1} \geq q_{k-1} m V_{k-1}\left(\alpha_{k-1}-V_{k-1}\right)\left(\alpha_{k-1}+V_{k-1}\right) \text {. }
$$

By inequality (37) and the comparison method [18] and Lemma 1, we establish the following lemma. The presented result provides the lower estimate that in turn establishes the locally lower cohesive property of (15).

Lemma 3: Let $V_{k-1}$ be the energy function defined in (18), $k \in I(1, \infty), t \in\left[t_{k-1}, t_{k}\right)$, and $z_{i}^{k-1}$ be a solution of the initial value problem defined in (15). Let $\rho_{k-1}(t)$ be the minimal solution [18] of a random initial value problem

$$
d u_{k-1}=q_{k-1} m u_{k-1}\left(\alpha_{k-1}-u_{k-1}\right)\left(\alpha_{k-1}+u_{k-1}\right) d t, \quad u_{k-1}\left(t_{k-1}\right)=u_{k-1},
$$

where $\alpha_{k-1}$ is as defined in (35). For each $V_{k-1}\left(z_{i}^{k-1}\right), i \in I(1, m)$, and $k \in I(1, \infty)$ satisfying the differential inequality (37) and $V\left(z_{i}^{k-1}\left(t_{k-1}\right)\right) \geq u_{k-1}$, it follows that the multicultural dynamic network (10) is lower cohesive on $\left[t_{k-1}, t_{k}\right]$ with probability 1 and

$$
V_{k-1}\left(z_{i}^{k-1}(t)\right) \geq \rho_{k-1}\left(t, t_{k-1}, u_{k-1}\right) .
$$

Proof: From inequality (37) and Lemma 1 and the imitating the outline of the proof of Lemma 2, it follows that

$$
V_{k-1}\left(z_{i}^{k-1}(t)\right) \geq \rho\left(t, t_{k-1}, u_{k-1}\right)
$$

provided that $V_{k-1}\left(z_{i}^{k-1}\left(t_{k-1}\right)\right) \geq u_{k-1}$. As the minimal solution of (38) is a lower bound, the network is lower cohesive almost surely. Moreover, a remark similar to Remark 1 establishes the locally stochastic mean and probability of (15).

We note that comparison differential equations (30) and (38) each have a unique solution process. Therefore the maximal and minimal solutions of (30) and (38) are the unique solutions of the respective random initial value problems.

\section{Long-term Behavior of Comparison Differential Equations and Invariant Sets}

To appreciate the role and scope of Lemmas 2 and 3, we seek to better understand both the behavior of the network on each interval $\left[t_{k-1}, t_{k}\right]$ and the long-term behavior of the network. For this purpose, for 
$k \in(1, \infty)$, we find the closed form solutions of the comparison random initial value problems (30) and (38). Moreover, we analyze the qualitative properties of the solutions to the comparison equations. Using the comparison method [18], we are able to establish, quantitatively, the behavior of the individual member cultural dynamic states on the interval $\left[t_{k-1}, t_{k}\right]$. Using this, we also establish the overall longterm behavior of both individual member cultural dynamic states in the network as well as multicultural network state as a whole.

Following the method of finding the closed form solution process of the initial value problem [19], the solution of (38) is represented by

$$
u_{k-1}\left(t, t_{k-1}, u_{k-1}\right)=\frac{u_{k-1} v}{\sqrt{u_{k-1}^{2}+\left(v^{2}-u_{k-1}^{2}\right) \exp \left[-2 v^{2} q_{k-1} m\left(t-t_{k-1}\right)\right]}} .
$$

As $z_{i}^{k}\left(t_{k}\right)=\left(1+\delta_{i}^{k-1}\right) z_{i}^{k-1}\left(t_{k}^{-}, t_{k-1}, x_{i}^{k-1}\right)$ for $k \in I(0, \infty)$, we seek to write the initial position $u_{k}$ in terms of $u_{0}$.

By squaring both sides and rearranging the terms, we can write the above as

$$
\frac{u_{k-1}^{2}\left(t, t_{k-1}, u_{k-1}\right)}{v^{2}-u_{k-1}^{2}\left(t, t_{k-1}, u_{k-1}\right)}=\frac{u_{k-1}^{2} \exp \left[2 v^{2} q_{k-1} m\left(t-t_{k-1}\right)\right]}{v^{2}-u_{k-1}^{2}} .
$$

We now set

$$
\begin{aligned}
& y_{k-1}\left(t, t_{k-1}, y_{k-1}\right)=\frac{u_{k-1}^{2}\left(t, t_{k-1}, u_{k-1}\right)}{v^{2}-u_{k-1}^{2}\left(t, t_{k-1}, u_{k-1}\right)} \\
& d y_{k-1}=\frac{2 u_{k-1}\left(t, t_{k-1}, u_{k-1}\right)\left[\left(v^{2}-u_{k-1}^{2}\left(t, t_{k-1}, u_{k-1}\right)\right)+2 u_{k-1}\left(t, t_{k-1}, u_{k-1}\right)\left(u_{k-1}^{2}\left(t, t_{k-1}, u_{k-1}\right)\right] d u_{k-1}\right.}{\left(v^{2}-u_{k-1}^{2}\left(t, t_{k-1}, u_{k-1}\right)\right)^{2}} \\
& =\frac{2 v^{2} u_{k-1}\left(t, t_{k-1}, u_{k-1}\right) d u_{k-1}}{\left(v^{2}-u_{k-1}^{2}\left(t, t_{k-1}, u_{k-1}\right)\right)} \\
& =\frac{2 v^{2} u_{k-1}\left(t, t_{k-1}, u_{k-1}\right)\left(q_{k-1} m u_{k-1}\left(t, t_{k-1}, u_{k-1}\right)\left(v^{2}-u_{k-1}^{2}\left(t, t_{k-1}, u_{k-1}\right)\right)\right) d t}{\left(v^{2}-u_{k-1}^{2}\left(t, t_{k-1}, u_{k-1}\right)\right)^{2}} \\
& =2 v^{2} q_{k-1} m\left(\frac{u_{k-1}^{2}\left(t, t_{k-1}, u_{k-1}\right)}{v^{2}-u_{k-1}^{2}\left(t, t_{k-1}, u_{k-1}\right)}\right) d t \\
& =\left(2 v^{2} q_{k-1} m\right) y_{k-1} d t \text {. }
\end{aligned}
$$

Therefore, on the interval $\left[t_{k-1}, t_{k}\right]$, the solution of Error! Reference source not found. is 


$$
y_{k-1}\left(t, t_{k-1}, y_{k-1}\right)=y_{k-1} \exp \left[2 v^{2} m q_{k-1}\left(t-t_{k-1}\right)\right], \quad y_{0}\left(t_{0}\right)=y_{0} .
$$

Let $\Delta t_{k}=t_{k}-t_{k-1}$. When $k=1$, the solution of $(44)$ on $\left[t_{0}, t_{1}\right)$ is

$$
\begin{array}{ll}
y_{0}\left(t, t_{0}, u_{0}\right) & =y_{0} \exp \left[2 v^{2} m q_{0}\left(t-t_{0}\right)\right] \\
y_{0}\left(t_{1}^{-}, t_{0}, u_{0}\right) & =y_{0} \exp \left[2 v^{2} m q_{0} \Delta t_{1}\right] \\
y_{1}\left(t_{1}\right) & =\left|1+\delta_{i}^{0}\right| y_{0} \exp \left[2 v^{2} m q_{0} \Delta t_{1}\right] .
\end{array}
$$

We assume that for $k-1 \in I(1, \infty)$, the solution of $(44)$ on $\left[t_{k-1}, t_{k}\right)$ is

$$
\begin{aligned}
y_{k-1}\left(t, t_{k-1}, y_{k-1}\right) & =\prod_{j=1}^{k-1}\left|1+\delta_{i}^{j-1}\right| y_{0} \exp \left[2 v^{2} m\left(\sum_{j=1}^{k-1} q_{j-1} \Delta t_{j-1}+\left(t-t_{k-1}\right)\right)\right] \\
y_{k-1}\left(t_{k}^{-}, t_{k-1}, u_{k-1}\right) & =\prod_{j=1}^{k-1}\left|1+\delta_{i}^{j-1}\right| y_{0} \exp \left[2 v^{2} m \sum_{j=1}^{k-1} q_{j-1} \Delta t_{j}\right] \\
y_{k}\left(t_{k}\right) & =\left|1+\delta_{i}^{k-1}\right| y_{k-1}\left(t_{k}^{-}, t_{k-1}, u_{k-1}\right) \\
& =\prod_{j=1}^{k}\left|1+\delta_{i}^{j-1}\right| y_{0} \exp \left[2 v^{2} m \sum_{j=1}^{k} q_{j-1} \Delta t_{j}\right] .
\end{aligned}
$$

Then for $k \in I(1, m)$, the solution of $(44)$ on $\left[t_{k}, t_{k-1}\right)$ is

$$
\begin{aligned}
y_{k}\left(t, t_{k}, y_{k}\right) & =y_{k} \exp \left[2 v^{2} m q_{k}\left(t-t_{k}\right)\right] \\
& =\prod_{j=1}^{k}\left|1+\delta_{i}^{j-1}\right| y_{0} \exp \left[2 v^{2} m\left(\sum_{j=1}^{k} q_{j-1} \Delta t_{j}+\left(t-t_{k}\right)\right)\right],
\end{aligned}
$$

and

$$
y_{k}\left(t_{k+1}^{-}, t_{k}, y_{k}\right)=\prod_{j=1}^{k}\left|1+\delta_{i}^{j-1}\right| y_{0} \exp \left[2 v^{2} m \sum_{j=1}^{k+1} q_{j-1} \Delta t_{j}\right],
$$

so

$$
\begin{aligned}
y_{k+1}\left(t_{k+1}\right) & =\left|1+\delta_{i}^{k+1}\right| y_{k}\left(t_{k+1}^{-}, t_{k}, y_{k}\right) \\
& =\prod_{j=1}^{k+1}\left|1+\delta_{i}^{j-1}\right| y_{0} \exp \left[2 v^{2} m \sum_{j=1}^{k+1} q_{j-1} \Delta t_{j}\right] .
\end{aligned}
$$

Therefore, using mathematical induction, it follows that for any $k \in I(1, \infty)$, 


$$
\begin{aligned}
y_{k-1}\left(t, t_{k-1}, y_{k-1}\right) & =\prod_{j=1}^{k-1}\left|1+\delta_{i}^{j-1}\right| y_{0} \exp \left[2 v^{2} m\left(\sum_{j=1}^{k-1} q_{j-1} \Delta t_{j-1}+\left(t-t_{k-1}\right)\right)\right] \\
y_{k}\left(t_{k}\right) & =\prod_{j=1}^{k}\left|1+\delta_{i}^{j-1}\right| y_{0} \exp \left[2 v^{2} m \sum_{j=1}^{k} q_{j-1} \Delta t_{j}\right]
\end{aligned}
$$

From the definition of $y_{k}$ and $(50)$, for $k \in I(1, \infty)$ and $t \in\left[t_{k-1}, t_{k}\right)$

$$
\begin{aligned}
u_{k-1}^{2}\left(t, t_{k-1}, u_{k-1}\right) & =\frac{v^{2} y_{k-1}\left(t, t_{k-1}, y_{k-1}\right)}{1+y_{k-1}\left(t, t_{k-1}, y_{k-1}\right)} \\
& =\frac{v^{2} \prod_{j=1}^{k-1}\left|1+\delta_{i}^{j-1}\right| u_{0}^{2}}{\prod_{j=1}^{k-1}\left|1+\delta_{i}^{j-1}\right| u_{0}^{2}+\left(v^{2}-u_{0}^{2}\right) \exp \left[-2 v^{2} m\left(\sum_{j=1}^{k-1} q_{j-1} \Delta t_{j}+\left(t-t_{k-1}\right)\right]\right]} \\
& =\frac{v^{2} u_{0}^{2}}{u_{0}^{2}+\left(v^{2}-u_{0}^{2}\right) \exp \left[-2 v^{2} m\left(\sum_{j=1}^{k-1} q_{j-1} \Delta t_{j}+\left(t-t_{k-1}\right)\right)\right] \prod_{j=1}^{k-1}\left|1+\delta_{i}^{j-1}\right|^{-1}}
\end{aligned}
$$

and

$$
u\left(t, t_{k}, u_{k}\right)=\frac{v u_{0}}{\left(u_{0}^{2}+\left(v^{2}-u_{0}^{2}\right) \exp \left[-2 v^{2} m\left(\sum_{j=1}^{k} q_{j-1} \Delta t_{j-1}+\left(t-t_{k}\right)\right)\right] \prod_{j=1}^{k-1}\left|1+\delta_{i}^{j-1}\right|^{-1}\right)^{\frac{1}{2}}}
$$

Further, for $k \in(1, \infty)$,

$$
u\left(t_{k}\right)=\frac{u_{0} v}{\left(u_{0}^{2}+\left(v^{2}-u_{0}^{2}\right) \exp \left[-2 v^{2} m \sum_{j=1}^{k+1} q_{j-1} \delta t_{j-1}\right] \prod_{j=1}^{k}\left|1+\delta_{i}^{j-1}\right|^{-1}\right)^{\frac{1}{2}}}
$$

By (53), taking the limit as $k \rightarrow \infty$, it follows that the initial positions $u_{k-1}$ will converge and

$$
\lim _{k \rightarrow \infty} u_{k-1}=v
$$

Further, by (52)

$$
\lim _{k \rightarrow \infty} u\left(t, t_{k-1}, u_{k-1}\right)=v \text {. }
$$

Therefore, taking the limit of the upper comparison solution $r\left(t, t_{0}, u_{0}\right)$ at $t \rightarrow \infty$, the long term behavior of is such that 


$$
\lim _{t \rightarrow \infty} r\left(t, t_{0}, u_{0}\right)=\eta
$$

where

$$
\eta=\limsup _{k \rightarrow \infty} \eta_{k-1}
$$

if it exists and

$$
\eta_{k-1}=\left(\frac{a_{k-1}}{q_{k-1}}+\frac{4 b_{k-1}(m-1) \sqrt{\frac{1}{2}} \exp \left[-\frac{1}{2}\right]+\beta_{k-1}^{2}(m-1) c_{k-1} \exp [-1]}{4 q_{k-1} m}\right)^{\frac{1}{2}} .
$$

Thus, if the limit superior $\eta$ exists, the solution process of (15) is globally upper cohesive in the a.s. sense on $[0, \infty)$.

Similarly, the limit of the solution of the lower comparison equation (38) as $t \rightarrow \infty$ is

$$
\lim _{t \rightarrow \infty} \rho\left(t, t_{0}, u_{0}\right)=\alpha
$$

where

$$
\alpha=\liminf _{k \rightarrow \infty} \alpha_{k-1}
$$

and

$$
\alpha_{k-1} \geq\left(\frac{a_{k-1}}{q_{k-1}}-\frac{4(m-1) b_{k-1} \sqrt{\frac{c_{k-1}}{2}} \exp \left[-\frac{1}{2}\right]+\beta_{k-1}^{2}(m-1) c_{k-1} \exp [-1]}{4 q_{k-1} m}\right)^{\frac{1}{2}} .
$$

Moreover, the solution process of (15) is globally lower cohesive a.s. on $\left[t_{0}, \infty\right)$.

Using the long term behavior of the comparison equations in conjunction with Lemmas 2 and 3, we establish the following theorem.

Theorem: Let the hypotheses of Lemmas 2 and 3 be satisfied. Then the network is locally cohesive in the almost surely on $\left[t_{k-1}, t_{k}\right)$ for $k \in I(1, \infty)$. If additionally $\eta$ exists and is finite, then the network is globally cohesive in the almost surely on $\left[t_{0}, \infty\right)$.

Proof: From Lemmas 2 and 3, 


$$
\rho_{k-1}\left(t, t_{k-1}, \rho_{k-1}\right) \leq V_{k-1}\left(z_{i}^{k-1}(t)\right) \leq r_{k-1}\left(t, t_{k-1}, r_{k-1}\right)
$$

with probability 1 . Moreover, as the solution to the upper comparison equation is bounded above by $\eta_{k-1}$ and the solution to the lower comparison equation is bounded below by $\alpha_{k-1}$, the network is cohesive almost surely. Suppose that $\eta$ exist and is finite. Then, we have

$$
\rho\left(t, t_{0}, u_{0}\right) \leq V\left(z_{i}\left(t, t_{0}, z_{i}^{0}\right)\right) \leq r\left(t, t_{0}, u_{0}\right)
$$

for $t \geq t_{0}$. As the solutions $\rho$ and $r$ are bounded, the network is globally cohesive with probability 1 .

\subsection{Invariant Sets}

In the case of the hybrid stochastic dynamical network, we can first consider the behavior of the solution process on the interval $\left[t_{k-1}, t_{k}\right)$. For $k \in I(1, \infty)$, let us denote

$$
r_{2}=\left(\frac{a_{k-1}}{q_{k-1}}-\frac{4(m-1) b_{k-1} \sqrt{\frac{c_{k-1}}{2}} \exp \left[-\frac{1}{2}\right]+\beta_{k-1}^{2}(m-1) c_{k-1} \exp [-1]}{4 q_{k-1} m}\right)^{\frac{1}{2}}
$$

and

$$
r_{1}=\left(\frac{a_{k-1}}{q_{k-1}}+\frac{4 b_{k-1}(m-1) \sqrt{\frac{c_{k-1}}{2}} \exp \left[-\frac{1}{2}\right]+\beta^{2}(m-1) c_{k-1} \exp [-1]}{4 q_{k-1} m}\right)^{\frac{1}{2}} .
$$

Further, let us define the following sets:

$$
\begin{cases}A_{k-1} & =B\left(0, r_{2}\right) \\ B_{k-1} & =B^{c}\left(0, r_{2}\right) \cap B\left(0, r_{1}\right) \\ C_{k-1} & =B^{c}\left(0, r_{1}\right)\end{cases}
$$

From the analysis developed in that section, we establish the following theorem for the solution on the interval $\left[t_{k-1}, t_{k}\right]$.

Theorem: Let the hypotheses of Lemmas 2 and 3 be satisfied. Then almost surely,

i. the set $A_{k-1} \cup B_{k-1}$ is conditionally invariant relative to $A_{k-1}$;

ii. the set $B_{k-1}$ is self-invariant; 
iii. the set $B_{k-1} \cup C_{k-1}$ is conditionally invariant relative to $C_{k-1}$.

Proof: Following the proof outlined in [3], the result follows directly.

By considering the limit as $k \rightarrow \infty$, we also establish the following result for the long-range invariant sets of (15).

For $k \in(1, \infty)$

$$
\lim _{k \rightarrow \infty} u\left(t_{k-1}\right)=\lim _{k \rightarrow \infty} u\left(t, t_{k-1}, u_{k-1}\right)
$$

for both the upper and lower comparison equations, then as $k \rightarrow \infty$

$$
\alpha \leq\left\|z_{i}^{k-1}\left(t_{k-1}\right)\right\| \leq \eta
$$

and

$$
\alpha \leq\left\|z_{i}^{k-1}\left(t, t_{k-1}, z_{i}^{k-1}\right)\right\| \leq \eta
$$

for sufficiently large $k \in(1, \infty)$. Thus, (15) exhibits long-range self-invariance for every member of the network.

In Section 6, we use numerical simulations to better understand the estimates and network behavior on the intervals $\left[t_{k-1}, t_{k}\right]$ for a finite number $k$.

\section{Numerical Simulations}

In this section, we consider numerical simulations for the multicultural dynamic network governed by the stochastic differential equation (15). We use a Euler-Maruyama [20-22] type numerical approximation scheme. We consider a network of six members, using the same initial position and varying the parameters $a_{k-1}, b_{k-1}$, and $\beta_{k-1}, k \in I(1, \infty)$. Further, we consider the case such that $\xi_{i j}^{k-1}(t)$ for $i, j \in I(1,6)$ is a one-dimensional Brownian motion process with mean of zero and variance of 1 over the interval $[0,1]$.

Often in a cultural network, events such as natural disasters, sudden political or economic changes, etc., can cause rippling effects in the cultural network. These changes can be characterized by the parametric changes in the stochastic differential equation (15). Therefore, we choose to simulate such a situation in the models in this section. Here, we choose 5 arbitrary times $t_{k}$ on the interval $(0,1)$ for which the model experiences an intervention on the dynamic. Further, for each $t_{k}, k \in I(1,5)$, we set $x_{i}^{k}\left(t_{k}\right)=\left(1+\delta_{i}^{k}\right) x_{i}^{k}\left(t^{-}\right)$, where $\delta_{i}^{k}$ is a constant for fixed $i$ and $k \in I(1,5)$, and consider the various scenarios based on changing the parameters $a_{k}, b_{k}$ and $\beta_{k}$. 
In order to consider the effects of changing the parametric quantity $a_{k-1}$, we consider various models for which $\beta_{k-1}=2, b_{k-1}=1, c_{k-1}=2$, and $q_{k-1}=1 / 7$ are held constant for $k \in I(1,5)$ and $a_{k}=a_{k-1}+1$, $a_{0}=2$. The plot of the position $z_{i}(t)$ for $t \in[0,1]$ is given in Figure 1 .

In order to consider the effects of changing the parametric quantity $b_{k-1}$, we consider the model for which $a_{k-1}=2, \beta_{k-1}=2, c_{k-1}=2$, and $q_{k-1}=1 / 7$ are held constant for $k \in I(1,5)$ and $b_{k}=b_{k-1}+1, b_{0}=1$. Figure 2 exhibits the simulated positions of the members $Z_{i}$.

In order to consider the effects of changing the parametric quantity $\beta_{k-1}$, we consider the model for which $a_{k-1}=2, b_{k-1}=1, c_{k-1}=2$, and $q_{k-1}=1 / 7$ are held constant for $k \in I(1,5)$ and $\beta_{k}=\beta_{k-1}+1$, $\beta_{0}=2$. In Figure 3 , we plot the positions of the members for $t \in[0,1]$.

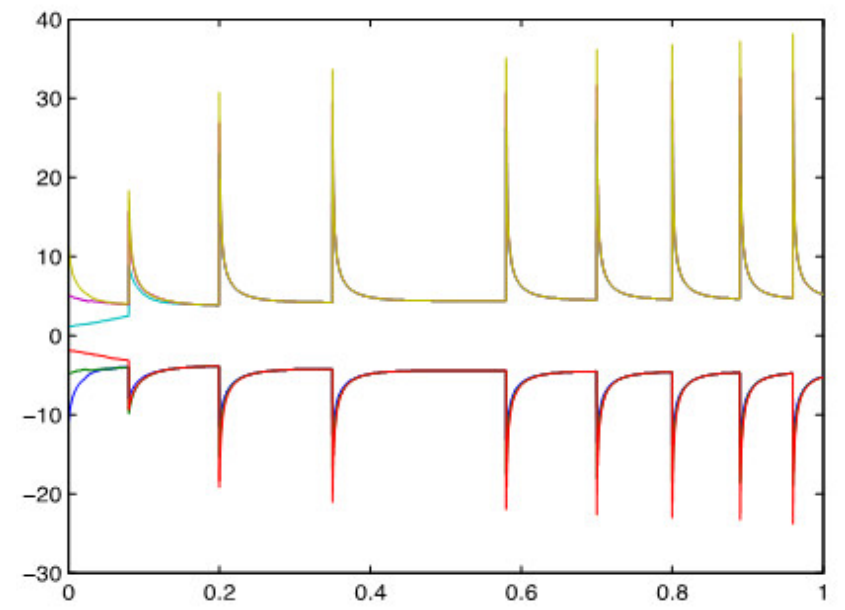

Figure 1: Euler-Maruyama approximation of the differential equation with six members and parameter ak=ak-1+1.

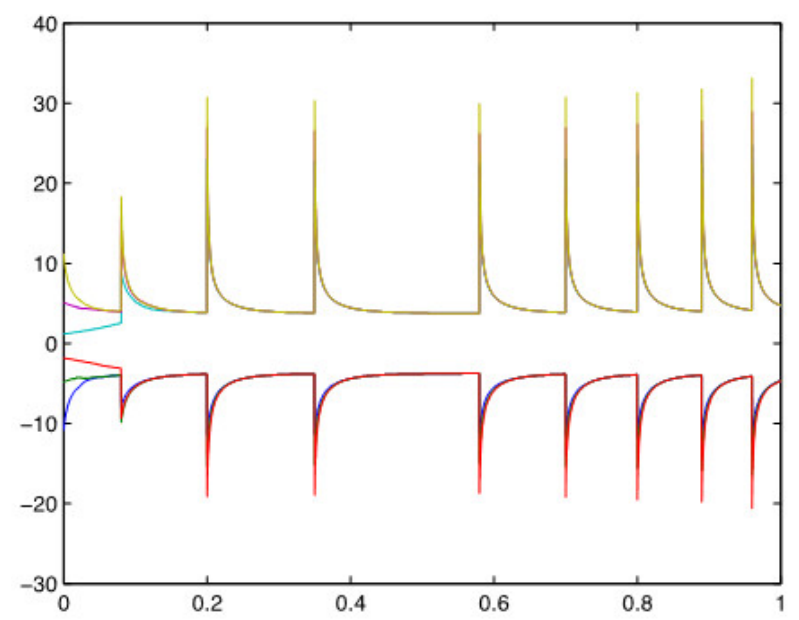

Figure 2: Euler-Maruyama approximation of the differential equation with six members and parameter bk=bk-1+1 
In order to consider the effects of a change in the parametric quantity $a_{k-1}$ and $\beta_{k-1}$, we consider the model for which $b_{k}=1, c_{k-1}=2$, and $q_{k-1}=1 / 7$ are held constant for $k \in I(1,5)$ and $a_{k}=a_{k-1}+1$, and $\beta_{k}=\beta_{k-1}+1, \beta_{0}=2$. The plot of the member's positions of the simulated network is given in Figure 4 .

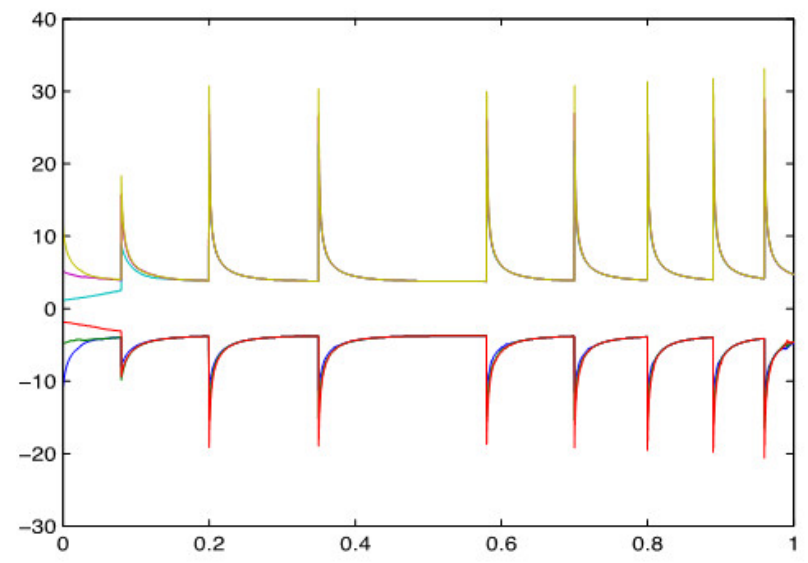

Figure 3: Euler-Maruyama approximation of the differential equation with six members and parameter $\beta k=\beta k-1+1$.

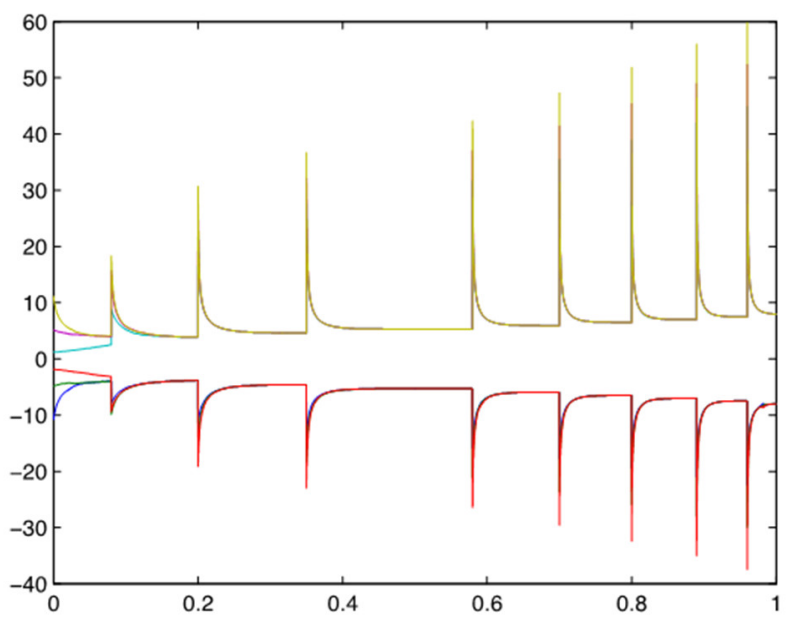

Figure 4: Euler-Maruyama approximation of the differential equation with six members and parameters $a k=a k-1+1, b k=b k-1+1$, and $\beta k=\beta k-1+1$.

\section{Conclusion}

Maintaining diversity while simultaneously fostering a sense of community membership, individual cultural identity, and cohesion is currently a goal among communities worldwide. It is important for members in a society to both feel as a part of the community in which they live and interact as well as feel free to embrace a strong sense of self and individuality. We seek to better understand the factors that play a role in obtaining such a balance by considering the impact of the repulsive and attractive forces influencing the multicultural network as in the previous work [2,3]. Attractive influences can be thought of as attributes that bring people to active membership within the group. Social acceptance, gaining social status, economic opportunity, career growth, common purpose and membership, personal development, 
and a sense of mutual respect, trust and understanding are examples of attractive influences within a social cultural network. Repelling forces are attributes that create some desire for individuals to leave or be less involved in the group or to preserve some personal identity from one other with their individual magnitude of inner repulsive force. A desire to retain a sense of individuality, economic or emotional cost, interpersonal conflict within the group, or disagreement with parts of the overall philosophies of the group are forces that may be considered as repulsive forces. The goal of the presented multicultural dynamic network is model the balance sought by members of the network in achieving these types of objectives. By doing so, we can consider the impact that policies and environmental factors may have on such a network.

By considering a hybrid dynamic model, we are able to better understand the impacts of outside influences that occur within community members and the cultural impacts such events have on the modeled cultural network. We have considered change based on the parameters that allow the perturbed multicultural dynamic network to remain cohesive while retaining a cultural state that is distinctive from the cultural state center of the network. We established qualitative and quantitative conditions that are computationally attractive and verifiable. We also conducted simulations of the multicultural network that exhibit the influence of the random perturbations and intervention processes as well as demonstrate the long-term behavior of the multicultural network. The presented results provide a tool for planning, performance, and implementations of policies and procedures within a social network.

We are interested in further exploring similar multicultural networks in the context of better understanding the relative cultural affinity $\left\|x_{i j}\right\|$ between members within the network and not just the cultural affinity between the cultural state of a member relative to the center of the network. The goal is to better understand the environmental factors that help foster a sense of individuality and diversity between all members within the network while maintaining a cohesive structure.

\section{ACKNOWLEDGEMENT}

The authors would like to acknowledge the research support by the Mathematical Sciences Division, US Army Research Office, Grant No. W911NF-15-1-0182.

\section{REFERENCES}

[1] Chandra, J. and G.S. Ladde, Collective behavior of multi-agent network dynamic systems under internal and external perturbations. Nonlinear Analysis: Real World Applications, 2010. 11(3): p. 1330-1344.

[2] Hilton, K.B. and G.S. Ladde, Deterministic Multicultural Dynamic Networks: Seeking a Balance between Attractive and Repulsive Forces. International Journal of Communications, Network and System Sciences, 2016. 9(12): p. 582-602.

[3] Hilton, K.B. and G.S. Ladde, Stochastic Multicultural Networks. Dynamical Systems and Applications, 2017. In Press. 
[4] Anabtawi, M.J., S. Sathannanthan, and G.S. Ladde, Convergence and stability analysis of large-scale parabolic systems under Markovian structural perturbations. International Journal of Applied Mathematics, 2000. 2(1): p. 57-85.

[5] Ladde, G.S. and B.A. Lawrence, Stability and convergence of large-scale stochastic approximation. International Journal of Systems Science, 1995. 26(3): p. 595-618.

[6] Ladde, G.S. and D.D. Siljak, Connective stability of large-scale stochastic systems. International Journal of Systems Science, 1975. 6(8): p. 713-721.

[7] Siljak, D.D., Large-scale Dynamic Systems: Stability and Structure1978, New York, NY: Elsevier NorthHolland.

[8] Acemoglu, D., et al., Opinion fluctuations and disagreement in social networks. Mathematics of Operations Research, 2013. 38(1): p. 1-27.

[9] DeGroot, M.H., Reaching a consensus. Journal of the American Statistical Association, 1974. 69(345): p. 118-121.

[10] Friedkin, N.E. Complex objects in the polytopes of the linear state-space. arXiv preprint arXiv:1401.5339, 2004. January 2014.

[11] Ma, H., Literature survey of stability of dynamical multi-agent systems with applications in rural-urban migration. American Journal of Engineering and Technology Research, 2013. 13(1): p. 131-140.

[12] Cao, Y., et al., An overview of recent progress in the study of distributed multi-agen coordination. IEEE Transactions on Industrial Informatics, 2013. 9(1): p. 427-438.

[13] $\mathrm{Hu}, \mathrm{H.-x.,} \mathrm{et} \mathrm{al.,} \mathrm{Group} \mathrm{consensus} \mathrm{in} \mathrm{multi-agent} \mathrm{systems} \mathrm{with} \mathrm{hybrid} \mathrm{protocol.} \mathrm{Journal} \mathrm{of} \mathrm{the} \mathrm{Franklin}$ Institute, 2013. 350: p. 575-597.

[14] Huang, M. and J.H. Manton, Coordination and consensus of networked agents with noisy measurements: stochastic algorithms and asymptotic behavior. SIAM Journal on Control and Optimization, 2009. 48(1): p. 131-161.

[15] Zhu, Y.-K., X.-P. Guan, and X.-Y. Luo, Finite-time consensus for multi-agent systems via nonlinear control protocols. International Journal of Automation and Computing, 2013. 10(5): p. 455-462.

[16] Axelrod, R., The Complexity of Cooperation: Agent-based Models of Competition1997, Princeton, NJ: Princeton University Press.

[17] Ladde, G.S., Hybrid Dynamical Inequalities and Applications. Dynamical Systems and Applications, 2005. 14: p. 481-514.

[18] Ladde, G.S. and V. Lakshmikantham, Random Differential Inequalities1980, New York, NY: Academic Press.

[19] Ladde, A.G. and G.S. Ladde, An Introduction to Differential Equations: Stochastic Modeling. Vol. 2. 2013, Hackensak, NJ: World Scientific. 
[20] Higham, D.J., An algorithmic introduction to numerical simulation of stochastic differential equations. SIAM Review, 2001. 43(3): p. 525-546.

[21] Higham, D.J. and P.E. Kloeden, Maple and Matlab for stochastic differential equations in finance, in Programming Languages and Systems in Computational Economics and Finance2002, Springer. p. 233269.

[22] Kloeden, P.E. and E. Platen, Numerical Solution of Stochastic Differential Equations1992, New York, NY: Springer-Verlag. 